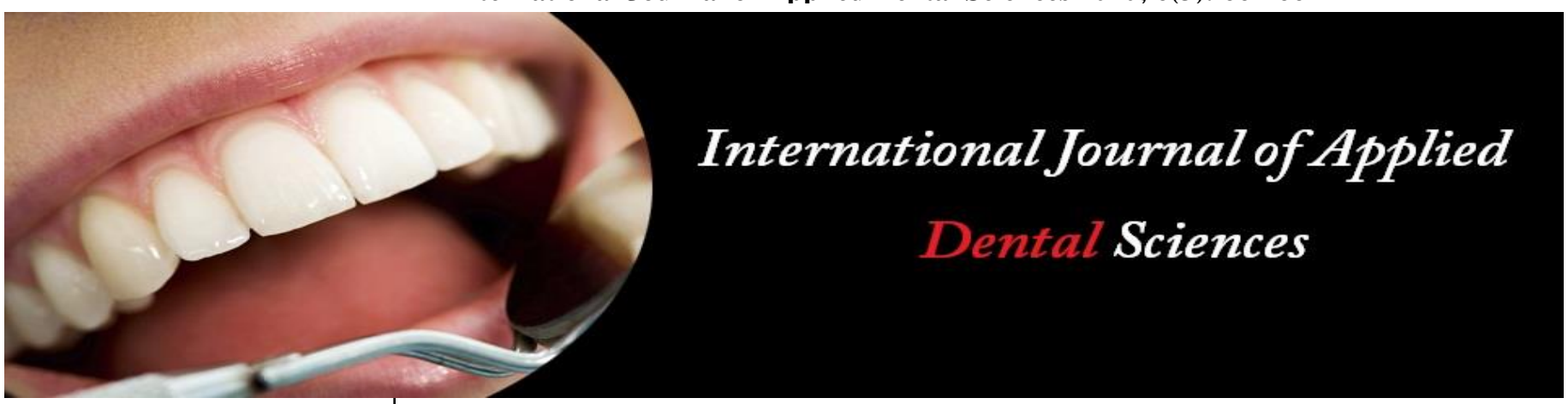

ISSN Print: 2394-7489

ISSN Online: 2394-7497

IJADS 2020; 6(3): 661-664

(C) 2020 IJADS

www.oraljournal.com

Received: 13-05-2020

Accepted: 15-06-2020

Dr. Omar Mohammad Al tammas

Post Graduate Student

Department of Conservative

Dentistry \& Removable

Prosthodontics Faculty of

Dentistry Hama University,

Hama, Syria

Dr. Hussein Ali Al Essa

Assistant Professor in

Department of Conservative

Dentistry \& Removable

Prosthodontics Faculty of

Dentistry, Hama University,

Syria

\section{In vitro study on reliability of digital scanning in duplicating removable partial dentures casts}

\author{
Dr. Omar Mohammad Al tammas and Dr. Hussein Ali Al Essa
}

DOI: https://doi.org/10.22271/oral.2020.v6.i3j.1021

\section{Abstract}

Aim: The main purpose of this study is to determine the dimensional accuracy of digital models as compared to that of study models and the reliability of digital scanning. The study aims to measure and compare distances between selected landmarks on study models and digital models and angles of cervical convergence of the abutment teeth.

Material and Methods: The study used partially edentulous study models of each of Kennedy's class I and III classifications. All the models were scanned using scanner and digitized on the computer. The physical models and digitized models were scanned and measured using various parameters and study designs. Measurements were conducted directly on study models and computer models using Altair Hyper mesh network version 7.0. The differences between the two seta of measurement were statistically analyzed using a suitable method ( $\mathrm{t}-$ test).

Results: The differences between measurement of distance on study models and digital models were found to be within the precision of $0,08 \mathrm{~mm}$ and 0,21 for the angle of cervical convergence of the abutment teeth. All these measurements were statistically slightly significant and well within the range of intra operator variations.

Conclusion: This study shows that dental cast can be scanned with the help of an accurate and sophisticated scanner and the scanned data can be transferred to the computer and used for the purpose of digital scanning.

Keywords: Computer aided design, dental cast, digital scanning, study model, three dimension

\section{Introduction}

For decades, removable partial dentures (RPDs) have been one of the treatment options among partially edentulous patients ${ }^{[1]}$ and sufficient retention is the key success of any removable prostheses' fabrication ${ }^{[2]}$.

The conventional procedure not only relies on the experience of a dental technician, but also incorporates multiple errors and inaccuracies in every step and often affects the prosthesis fit. A poor-fitting prosthesis may lead to pain and discomfort which further cause patient dissatisfaction. It also increases the laboratory and dentist's cost and chair time ${ }^{[3]}$. With continuous developments over several years, present-day technological advancements allow the use of different systems with computer-aided design/computer-aided manufacture (CAD/CAM) technology for the fabrication of removable dentures, including milling and rapid prototyping (RP) ${ }^{[4]} \mathrm{CAD} / \mathrm{CAM}$ technology refers to digital design and manufacture. CAD software recognizes thegeometry of an object while CAM software is used for the manufacture. The CAD/CAM manufacturing process can either include additive (RP) or subtractive manufacturing (computer numerical control [CNC] machining; milling). RP has been used for industrial purposes and was developed from CAD/CAM technology ${ }^{[5,6]}$, digital dentistry that incorporates computer-controlled components is playing an important role in dental prosthesis fabrication. The three-dimensional computer-aided design and computeraided manufacturing (CAD/CAM) technology has been introduced in fixed dental prostheses (FDPs) since 1980s. Instead of using a conventional impression technique, the digital impression is taken by either intraoral or laboratory scanner is used to capture the marginal detail and tooth structure. Any kind of indirect restorations can be then virtually designed using a specialized software and fabricated by means of a subtractive manufacturing ${ }^{[7]}$.
Dr. Omar Mohammad Al tamma

Post Graduate Student

Department of Conservative

Dentistry \& Removable

Prosthodontics Faculty of

Dentistry Hama University,

Hama, Syria 
The major advantage of a digital scanning is the capability of direct data acquisition from a prepared tooth in real time. It eliminates discomfort that patients may experience from a conventional impression procedure. It also minimizes cost, time and storage space for impression materials, disinfectants and gypsum models. Besides, the digital images can be stored indefinitely with good quality and achievability. However, some factors such as experience of dental technicians, requirement of implant-specific scan body, digital alteration of the occlusion, difficulty of scanning path should be taken into consideration ${ }^{[8]}$. Not only applicable in fixed dental prostheses, CAD/CAM also has potential to fabricate customized removable dental prosthesis by means of additive manufacturing through rapid prototyping ${ }^{[9]}$.

The evolution of the CAD/ CAM technology decreases the duration of prosthesis manipulation and provide superior functional and esthetic outcomes. Also changes of the prosthesis volume and/ or shape is reduced or eliminated in this approach compared to the conventional procedures. Thus, the produced prosthesis adheres tightly to the tissue and uniformly transferring loads on it ${ }^{[10]}$. Furthermore, it permits easy duplication of the denture and manufacture of new one using stored digital data ${ }^{[11]}$. The main benefits of introducing CAD/CAM in the fabrication of RPD framework involve automatic determination of a proposed path of insertion, the immediate elimination of undesirable undercuts, and the equally rapid identification of desirable undercuts. Not only is it a time saver, but also the CAD/CAM technology delivers inherent repeatability, which may assist in decreasing human errors and enhance quality control in the dental laboratory ${ }^{[12]}$. The purpose of this study was to verify the dimensional accuracy of the digitized 3D dental cast and reliability of surveying the same, which may eventually enable the prosthodontics procedures to be carried out digitally.

\section{Materials and Methods}

The research sample included 20 casts of partial edentulous, where the sample was randomly divided into two groups:

The first group: 10 casts included the first class of Kennedy classification

The second group: 10 casts included the third class of Kennedy classification. All study models were scanned using computerized digital scanning and digitization on the computer, then measurements were made on both studied and digital models using different criteria. The measurements were made directly on study models and digital models using special tools and programs, then the differences between the two measurements were statistically analyzed using appropriate methods.

The following standards were chosen to determine dimensional accuracy and reliability of digital scanning:

\footnotetext{
Measuring the linear distance between the predetermined points on the surface of the models: Where specific points were identified on study models and the distance between these points was measured using a digital ruler, and these points were also read and measurements made between them on digital models and recorded on the computer, Note that the previous measurements were repeated three times to avoid errors during the measurement process.
}

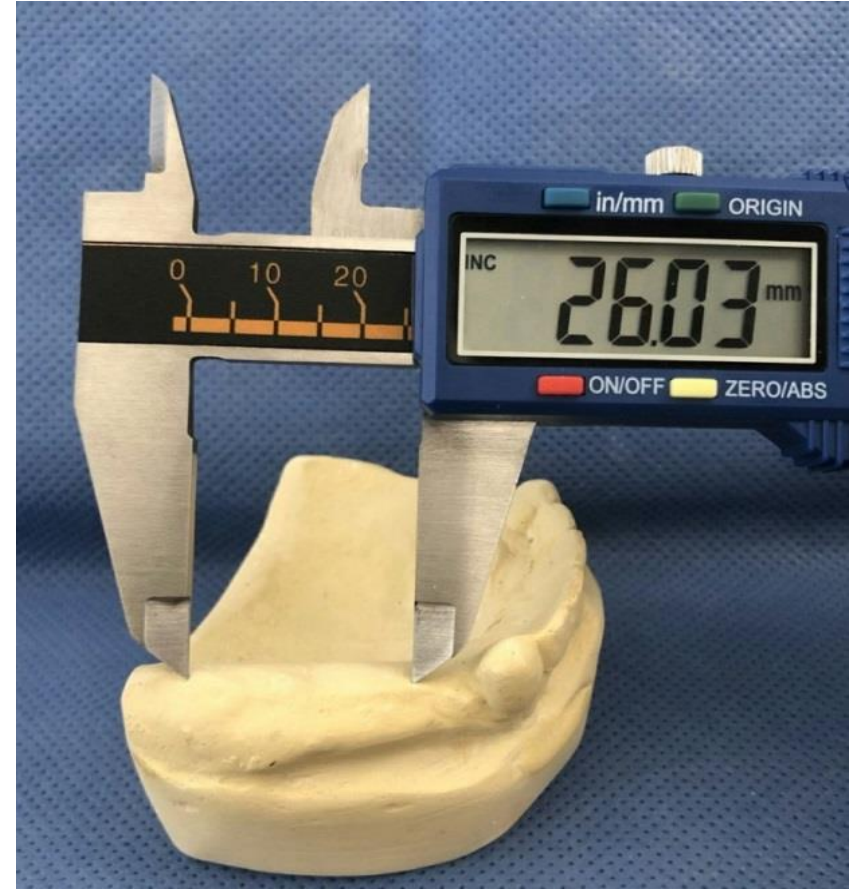

Fig 1: Measuring the distance between the two points using the digital ruler

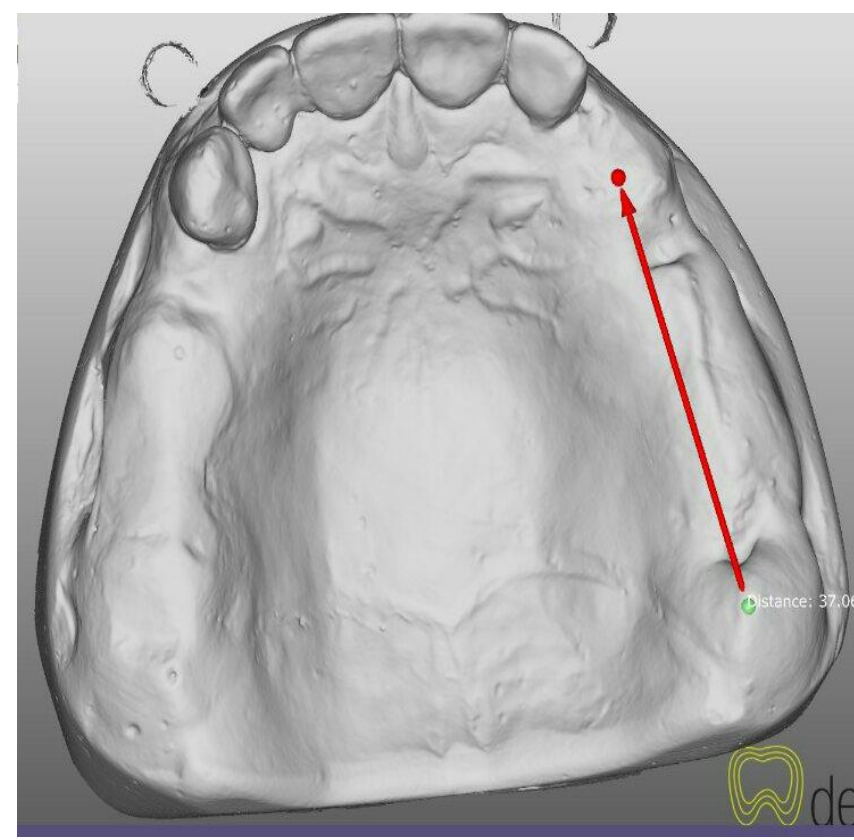

Fig 2: Reading holes on the CAD-CAM program and performing the measurement

Determination of angles of cervical convergence of the abutment teeth: Due to the difficulty of performing the measurement of the angles of the abutment teeth directly on the study models, three points were chosen on the gypsum casts that represent the angle of abutment teeth and through a mathematical relationship cosine of these angles was calculated and then the angle represented by the value of the cosine was obtained through a special program and this was also read Points on digital models, angles measured and recorded on a computer. 


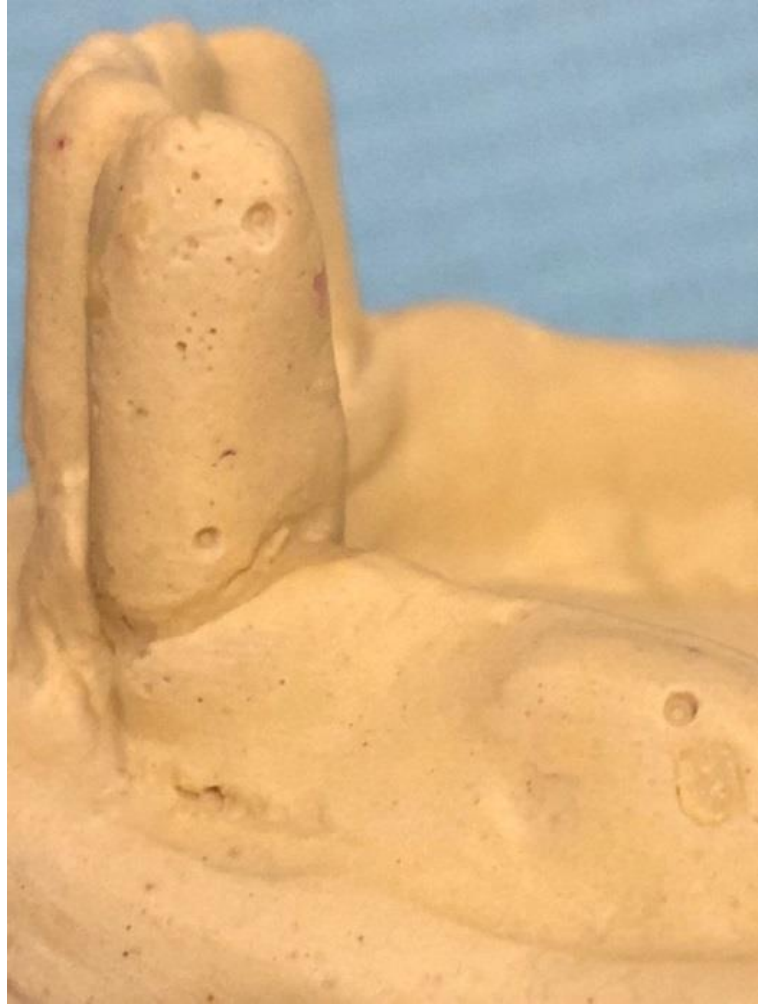

Fig 3: Identifying three holes representing angles of cervical convergence of the abutment tooth

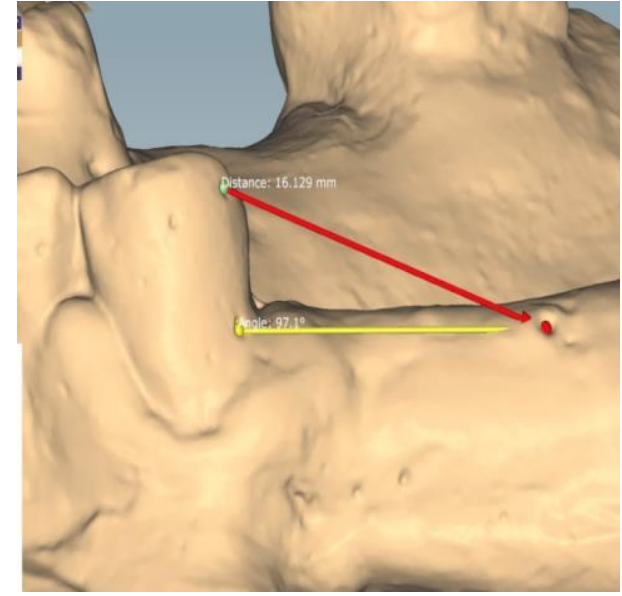

Fig 4: Measurement of angle of cervical convergence of the abutment tooth on CAD-CAM program

Results: The following results appeared in our current study conditions:

\begin{abstract}
Measurement of distance between predetermined points on the surface of the models: At a confidence level of $95 \%$, the distance measurement on the CAD-CAM program was greater than that of the gypsum cast, with an average mean of $0.08 \mathrm{~mm}$, but this statistically significant difference was not significant $(\mathrm{p}=0,163)$.
\end{abstract}

Table 1: $t$-test for correlated samples to study the difference between linear distance measurement on the gypsum casts and the CAD / CAM program

\begin{tabular}{|c|c|c|c|c|c|c|}
\hline \multirow{2}{*}{ p value } & \multirow{2}{*}{ t value } & \multicolumn{2}{|c|}{ The confidence interval is $95 \%$ of the average difference } & \multirow{2}{*}{$\begin{array}{l}\text { standard } \\
\text { deviation }\end{array}$} & \multirow{2}{*}{$\begin{array}{l}\text { Arithmetic average } \\
\text { of difference }\end{array}$} & \multirow{2}{*}{ variable } \\
\hline & & maximum & minimum & & & \\
\hline 0.163 & 1.452 & 0.19 & -0.03 & 0.24 & 0.08 & distances \\
\hline
\end{tabular}

2 - Determination of angles of cervical convergence of the abutment teeth: At a confidence level of $95 \%$, the measure of angles of cervical convergence of the abutment teethon the
CAD-cam program was greater than that of the gypsum example, with an average of $0.21 \mathrm{~mm}$, but this statistically significant difference was not significant $(\mathrm{p}=0,076)$.

Table 2: t-test for correlated samples to study the difference between angles of cervical convergence of the abutment teethmeasurement on the gypsum casts and the CAD / CAM program

\begin{tabular}{|c|c|c|c|c|c|c|}
\hline \multirow{2}{*}{ p value } & \multirow{2}{*}{$\mathbf{t}$ value } & \multicolumn{2}{|c|}{$\begin{array}{c}\text { The confidence interval is } \\
\text { 95\% of the average difference }\end{array}$} & $\begin{array}{c}\text { standard } \\
\text { deviation }\end{array}$ & $\begin{array}{c}\text { Arithmetic average } \\
\text { of difference }\end{array}$ & variable \\
\cline { 3 - 5 } & & maximum & minimum & & 0.21 & $\begin{array}{c}\text { angles of cervical convergence } \\
\text { of the abutment teeth }\end{array}$ \\
\hline 0.076 & 1.877 & 0.45 & -0.02 & 0.51 & 0.21 \\
\hline
\end{tabular}

\section{Discussion}

Computer aided design and computer aided machining techniques have been successfully introduced in the field of fixed partial denture ${ }^{[13]}$ and maxillofacial technology ${ }^{[14]}$ over a number of years. The development and evaluation of these advances continue. Advantages of such system have been well documented, and may well eventually become the "next generation" method of fabrication [15]. digital surveying of scanned cast enables a pattern for a metal framework to be fabricated on computer screen according to the principles of RPD Various methods were used for imaging dental hard structure such as holography, Moiré topography, Ppotostereometry, laser scanner etc ${ }^{[16]}$. For digital surveying, the digitized 3D image should be an accurate/reliable reproduction of the dental cast. If not, the clinical results will be affected ${ }^{[17,18]}$.

This study, therefore, was undertaken to determine the dimensional accuracy of digital models as compared to conventional study models. Study models and digital models of Kennedy's class I and class III partially edentulous situations were used. The main reason behind using Kennedy's classification is that, it permits immediate visualization of partially edentulous arch. It also permits a logical approach to the problem of design and makes possible the application of sound principle of partial denture design ${ }^{[19}$, 20].

For the purpose of determining the dimensional accuracy and the reliability of electronic surveying the following two criteria were used:

1. Measurement of the distance between predetermined points on the surface of the models.

2. Determination of angle of cervical convergence of the abutment teeth.

Some slight differences appeared in the measurement of linear distances between the predetermined points on both gypsum models and digital models, where the linear distances measured on the CAD CAM program ranged between 5.66- 
$37.12 \mathrm{~mm}$ and an average mean of $22.15 \mathrm{~mm}$, while on the gypsum models ranged between $5.50-36.87 \mathrm{~mm}$ and an average Mathematical calculation $22.07 \mathrm{~mm}$ When testing t to study the difference between linear distance measurements on the gypsum example and the cad cam program at a $95 \%$ confidence level, the results showed that the measurement of linear distances on the cad cam program is greater than on the gypsum example with an arithmetic mean of $0.08 \mathrm{~mm}$, but this difference was not significant Statistically $(P=0,163)$, Measurements of angles of cervical convergence of the abutment teeth on the cad cam program ranged between $.5079 .50-117.20^{\circ}$ and an average of $97.63^{\circ}$ while on the gypsum example ranged between $79^{\circ}-118^{\circ}$ and with an average score of $97.41^{\circ}$. And when testing t to study the difference between measuring the stent angles of the stent on the gypsum model and the cad cam program at $95 \%$ confidence level. The results showed that the measurement of angles on the CADCAM program is greater than that of the gypsum example, with an average of 0.21 , but this difference was not statistically significant $(\mathrm{P}=0.076)$.

The differences between the manual measurements and measurements made on the 3D image were slightly statistically significant and were, in fact, well within the range of intra operator variation. When the measurements are made on $3 \mathrm{D}$ digital image operators variation plays a role because the operator has to click on the points to be measured.

\section{Conclusion}

From this study we can conclude that the dental cast can be scanned with the help of an accurate and sophisticated scanner and the digital model used for the purpose of diagnosis, treatment planning and storage for future use. The dimensional accuracy of the digital models when compared to the study models was statistically significant Therefore, digital/ electronic surveying is a reliable method which can be accomplished by locating the height of contour and guide planes measurements as the digital model can be viewed from various angles and positions.

\section{References}

1. Frank RP et al., Relationship between the standards of removable partial denture construction, clinical acceptability, and patient satisfaction. The Journal of prosthetic dentistry. 2000; 83(5):521-527.

2. Shams A et al., Patient satisfaction and complication rates after delivery of removable partial dentures: A 4year retrospective study. SRM Journal of Research in Dental Sciences. 2015; 6(4):225.

3. Rudd RW, Rudd KD. A review of 243 errors possible during the fabrication of a removable partial denture: part I. The Journal of prosthetic dentistry, 2001; 86(3):251261.

4. Lima JMC et al., Removable partial dentures: use of rapid prototyping. Journal of Prosthodontics. 2014; 23(7):588-591.

5. Giacomo GAD et al., Clinical application of stereolithographic surgical guides for implant placement: preliminary results. Journal of periodontology. 2005; 76(4):503-507.

6. Sarment DP, Sukovic P, Clinthorne N. Accuracy of implant placement with a stereolithographic surgical guide. International Journal of Oral \& Maxillofacial Implants, 2003, 18(4).

7. Miyazaki T et al., A review of dental CAD/CAM: current status and future perspectives from 20 years of experience. Dental materials journal. 2009; 28(1):44-56.

8. Alghazzawi TF. Advancements in CAD/CAM technology: Options for practical implementation. Journal of prosthodontic research, 2016; 60(2):72-84.

9. Abduo J, Lyons K, Bennamoun M. Trends in computeraided manufacturing in prosthodontics: a review of the available streams. International journal of dentistry, 2014.

10. Persson AS et al. Digitization of simulated clinical dental impressions: virtual three-dimensional analysis of exactness. Dental materials. 2009; 25(7):929-936.

11. El-Khamisy N et al., Digital Versus Conventional Design For Mandibular Distal Extension RPD: A Study of Passivity of RPD Components and Principal Abutment Alveolar Bone Height Changes.

12. Campbell SD et al., Removable partial dentures: The clinical need for innovation. The Journal of prosthetic dentistry. 2017; 118(3):273-280.

13. Rudolph H, Quaas S, Luthardt R. Matching point clouds: limits and possibilities. International journal of computerized dentistry. 2002; 5(2, 3):155-164.

14. Kuroda T. et al., Three-dimensional dental cast analyzing system using laser scanning. American Journal of Orthodontics and Dentofacial Orthopedics. 1996; 110(4):365-369.

15. Reich $\mathrm{S}$ et al., Measurement of cement thickness under lithium disilicate crowns using an impression material technique. Clinical oral investigations. 2011; 15(4):521526.

16. Keating AP et al., A comparison of plaster, digital and reconstructed study model accuracy. Journal of orthodontics. 2008; 35(3):191-201.

17. Nan X et al., Clinical application of three-dimensional spiral CT in the complex maxillofacial fractures. Hua xi kou qiang yi xue za zhi= Huaxi kouqiang yixue zazhi= West China journal of stomatology, 2001; 19(6):372-374.

18. Brosky ME et al., Laser digitization of casts to determine the effect of tray selection and cast formation technique on accuracy. The Journal of prosthetic dentistry. 2002. 87(2):204-209.

19. Henderson D et al., McCracken's removable partial prosthodontics. CV Mosby Company, 1985.

20. Rohit P, Shetty S, Satish B. Electronic survey: An invitro study on reliability of the technique. The Journal of Indian Prosthodontic Society, 2009; 9(2):84. 